\title{
The safety and efficacy of IV sedation using propofol target-controlled infusions
}

\author{
Propofol target-controlled infusions for sedation - a safe technique for the non-anaesthetist?
}

M. R. Blayney, J. D. Ryan and A. F. Malins Br Dent J 2003; 194: 450-452

\section{Objective}

As an alternative to general anaesthesia for dentistry, attention has been focused on other, apparently safer, methods of pain and anxiety control. We have undertaken a study to evaluate the safety and efficacy of intravenous sedation using propofol targetcontrolled infusions.

\section{Design}

We describe a retrospective observational analysis of propofol conscious sedation as an adjunct to local anaesthesia for patients undergoing simple or surgical exodontia. All the patients were assessed, selected and treated according to standardized hospital sedation protocols. Experienced anaesthetists used a standard regimen, with ECG, pulse oximetry and non-invasive blood pressure monitoring. A standard sedation record was completed for each patient. The initial target plasma propofol concentration was set at $1.5 \mathrm{mg} \mathrm{ml}^{-1}$, adjusted thereafter to achieve the desired level of sedation. Any adverse events were recorded.

\section{Setting}

Birmingham Dental Hospital.

\section{Subjects}

Three hundred consecutive sedation episodes in adult dental phobic patients requiring exodontia under local anaesthesia.

\section{Results}

Sedation and treatment were satisfactorily completed in 297 patients. The mean target propofol concentration required was 2.1 $\mathrm{mg} \mathrm{ml}^{-1}\left(\mathrm{SD}=0.47 \mathrm{mg} \mathrm{ml}^{-1}\right.$, range $\left.1-4 \mathrm{mg} \mathrm{ml}^{-1}\right)$. General anaesthesia occurred in two cases (target concentration (TC) $2 \mathrm{mg}$ $\mathrm{ml}^{-1}$ ), over sedation in 11 cases (TC $1-3 \mathrm{mg} \mathrm{ml}^{-1}$ ), and transient hypoxaemia in 7 cases (TC $1.8-2.5 \mathrm{mg} \mathrm{ml}^{-1}$ ).

\section{Conclusion}

Intravenous sedation with target-controlled propofol infusions is effective. However, significant adverse effects may occur at target concentrations below $2.1 \mathrm{mg} \mathrm{ml}^{-1}$. Only anaesthetists working in an appropriate environment should practise this technique.

\section{IN BRIEF}

- Target-controlled infusions using propofol, an intravenous general anaesthetic agent, can be effectively used in sub-anaesthetic doses to produce conscious sedation for dentistry.

- Propofol does not demonstrate the margin of safety required to satisfy the General Dental Council definition of a sedative agent suitable for use by dentists.

- Propofol should be administered by trained anaesthetists working in an appropriate clinical environment.

\section{COMMENT}

This is an interesting paper which adds considerably to the debate surrounding new methods for pain and anxiety control in dentistry and in the wider field of medicine generally. The authors have collected data on around 300 patients who were treated using a target-controlled infusion of propofol, the dose of which was controlled by the anaesthetist in charge of the patient.

Treatment was completed in almost all cases. However, there were an unacceptable level of adverse events as defined in the paper of nearly $10 \%$. All of those working in this area, including the authors, would admit that this is unacceptable. The adverse events included over-sedation, reduced respiration rate and decreased oxygen saturation. The General Dental Council in their widely accepted definition of conscious sedation state that verbal contact must be maintained at all times and anything falling outside this definition is not conscious sedation as it is defined in the UK.

So, where does propofol sedation go from here? This is just one article in an increasing literature on the subject and the authors make a number of perhaps controversial conclusions including the requirement for all patients to starve before propofol sedation, and propofol sedation not fitting into the General Dental Council's definitions for requirements for conscious sedation. They go on to say that they would advocate only anaesthetists practising this technique. This may well be true for the technique outlined in this paper.

However, there is significant work going on in a number of fields including patient controlled infusions of propofol and a number of papers, principally in the wider medical literature, have started their propofol concentrations at a lower level and thereby appear to avoid some of the adverse effects mentioned.1,2,3

This retrospective analysis is helpful but must be read along with other literature on the subject. ${ }^{4}$ If we are to move patient care forward from intravenous bolus doses of midazolam which some have suggested, particularly in endoscopy, has an unacceptable mortality rate ${ }^{5}$ then we require randomised controlled trials of significant power in this area.

J. A. Leitch, Clinical Lecturer, Glasgow Dental Hospital and School

1. Irwin M G, Thompson N G N, Kenny C. Patient-maintained propofol sedation. Anaesth 1997; 52: 525-530.

2. Murdoch J A C, Kenny G N C. Patient-maintained propofol sedation as premedication in day-case surgery: assessment of a target-controlled system. Br J Anaesth 1999; 82: 429-431.

3. Gillham M J, Hutchinson R C, Cater R, Kenny, G N. Patient-controlled sedation for ERCP using a target-controlled infusion of propofol: a pilot study. Gastrointest Endosc 2001; 54: 14-17.

4. Leitch J A, Sutcliffe N, Kenny G N C. Patient-maintained sedation for oral surgery using a target-controlled infusion of propofol - a pilot study Br Dent J 2003 194; 1 : 43-45.

5. Quine M A, Bell G D, McCloy R F, Charlton J E, Devlin H B, Hopkins A. Prospective audit of upper gastrointestinal endoscopy in two regions of England: safety, staffing and sedation methods. Gut 1995; 95: 462-467. 